\title{
OVERVIEW OF NEW LIGHT SOURCES
}

\author{
M.S. de Jong*, Canadian Light Source, \\ University of Saskatchewan, Saskatoon, SK S7N 5C6, Canada
}

\begin{abstract}
Over the past few years, the use of synchrotron light sources has continued to grow rapidly. Several new facilities are in various phases of development. This paper provides an overview of the new facilities that have been proposed, have started construction, or are being commissioned throughout the world. Several trends in the design and specification of these facilities are reviewed, and possible future directions of light source evolution are examined.
\end{abstract}

\section{INTRODUCTION}

Over the past two decades there has been a huge increase in both the number of users and in the number of scientific disciplines using synchrotron light. For example, Figure 1 shows the growth in the Canadian synchrotron user community over the past sixteen years. Similarly, Figure 2 shows the growth in the international user community over the past decade.

While most of the initial synchrotron light applications were in physics and inorganic chemistry, today the range of applications includes:

- X-ray Absorption Fine Structure (XAFS) and related analysis techniques in chemistry, permitting chemical speciation analysis with remarkable sensitivity and spatial resolution;

- Protein Crystallography, permitting the determination of molecular structure of many complex bio-molecules of significance to biochemistry and the pharmaceutical industry;

- Diffraction-limited imaging in the infra-red region, permitting exceptionally interesting analysis in biology and medicine;

- New x-ray imaging techniques allowing much finer spatial and density resolution for medical diagnostic applications;

- XAFS and small molecule crystallography for environmental contamination analysis permitting the detection of heavy-metal contamination in soil samples, as well as the chemical form of the contamination.

This abbreviated list just begins to outline the many applications of synchrotron light experiment techniques that are having a dramatic impact on many areas of science. Today, research using synchrotron light is making a prominent contribution to all science disciplines. This growth now results in a significant

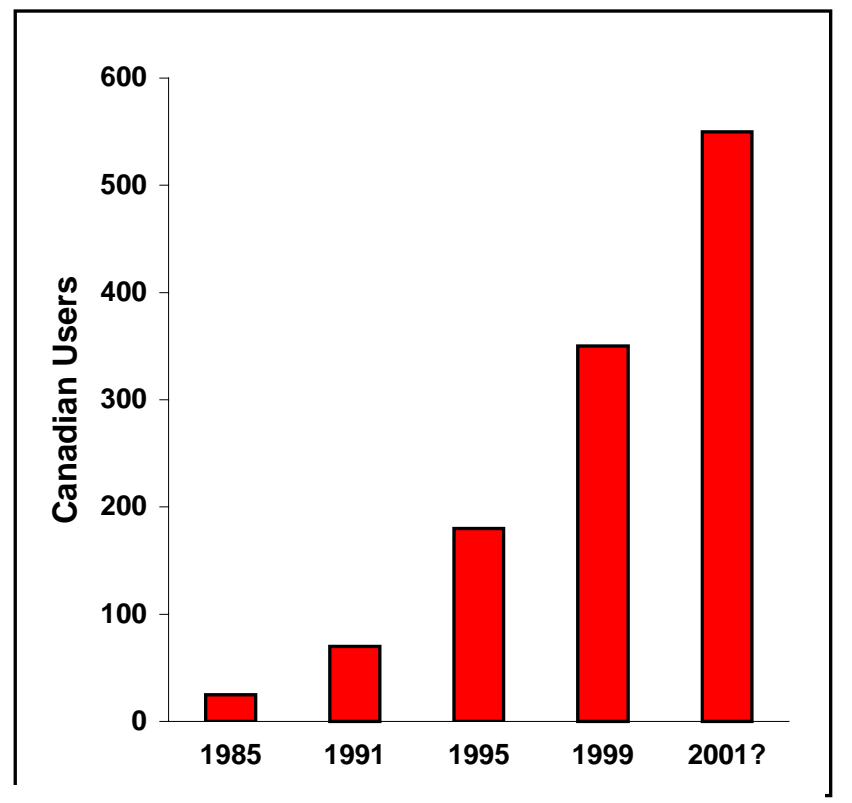

Figure 1: Growth in Canadian synchrotron user community over the past sixteen years

demand for additional national synchrotron light facilities

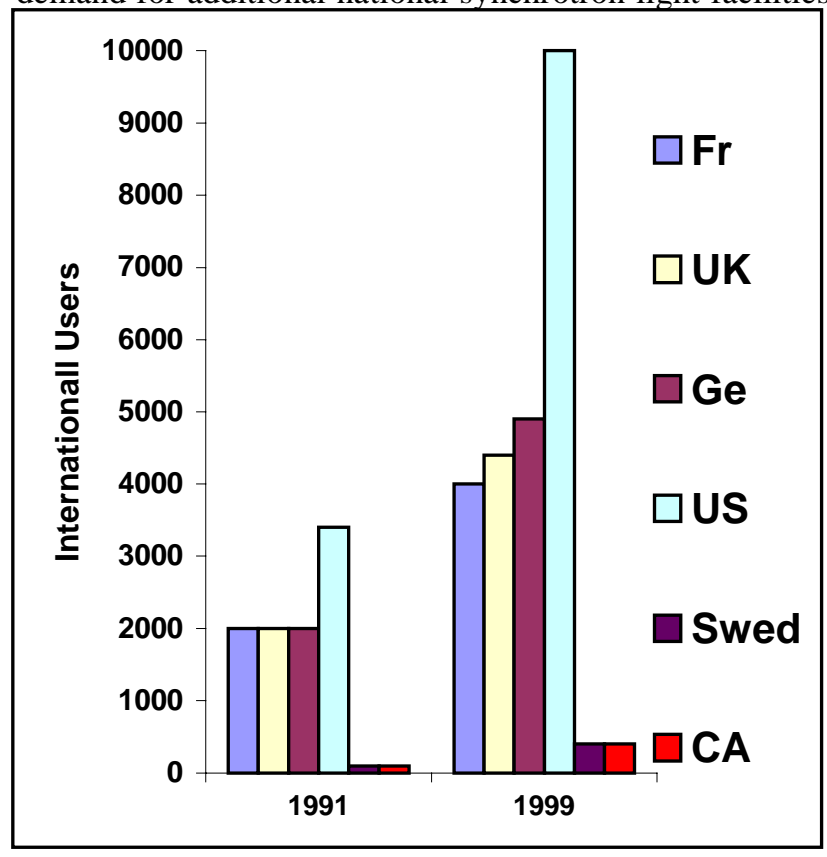

Figure 2: Growth in the international synchrotron user community over the past decade

*dejongm@cls.usask.ca 
to serve the scientific community within major countries as well as the broader international community. At the same time, there is growing interest in exploiting industrial applications of synchrotron light. Several facilities have started (or soon will start) fee-for-service business targeted at industry. A wide variety of companies are interested in these developments. In Canada, these companies include pharmaceutical, chemical analytical service, and mining companies.

A secondary consequence of this growth is that, by now, the majority of synchrotron users are non-physicists. These users are often more demanding of, and less sympathetic to, the facility design and operation staff.

\section{USER PHOTON REQUIREMENTS}

From an accelerator and facility designer's perspective, it is useful to examine the photon requirements of the present and future user community, especially to determine where these are different from past assumptions.

Certainly the demand for higher flux or brightness continues, although this demand most frequently arises from users on bending magnet beam-lines. The brightness of modern high-performance beam-lines using undulator radiation can be so high that samples suffer significant radiation damage in the time taken to acquire data. On new third generation facilities, the initial user demand is for insertion device beam-lines. Usually, only when these lines are no longer allocated or available will users accept a source point from a bending magnet.

Today, user demands often reflect the need for greater flexibility, stability and quality. The lower electron beam emittance of third generation sources naturally leads to lower photon beam emittance, often requiring sub-micron position stability in critical beam-line components.

The growing interest in circular and elliptical polarized $\mathrm{x}$-ray radiation for a variety of experiments leads to demands for the ability to change or reverse the polarization rapidly, at a frequency greater $10 \mathrm{~Hz}$ if possible. Other experiments require changing undulator photon energy within seconds. However, the polarization or the energy changes must be achieved without significant change in the electron or photon beam position.

Similarly, the growing interest in using the very low energy infrared radiation results in other stability challenges for the accelerator designer. Often the infrared experiments use Fourier transform techniques, with the result that small coherent oscillations in the infrared beam intensity can become a significant noise problem in spectra. A typical requirement in these experiments is that the intensity stability is within $0.01 \%$ in the frequency range from $1 \mathrm{~Hz}$ to $100 \mathrm{kHz}$. This requirement again presents a significant challenge to facility designers because of the huge number of electron storage ring phenomena that could lead to intensity variations in this frequency range.
User expectations of the facility reliability have increased significantly as well, with most new facilities having a target of more than 5000 hours per year of scheduled operation, with an availability of more $95 \%$. However, the experiment time for many users is allocated in quite short time periods, often for just a few shifts, so that any facility unavailability can affect a significant number of users. Thus, the average length of unscheduled outages should be less than a single shift.

One other significant requirement is the desired photon energy. In most cases, the experimental technique used and the materials studied determine the photon energy range. As synchrotron-based science has developed, it is becoming more clear that few techniques require photon energy over $40 \mathrm{keV}$, and most experiments are using an energy under $20 \mathrm{keV}$.

In addition to these requirements from users, there is the usual additional requirement from funding agencies to reduce the overall facility cost as far as possible, consistent with meeting the technical requirements. For current third generation sources, a reasonable target is that less than $50 \%$ of the total facility capital costs (including a full complement of beam-lines) should be required for the conventional building and accelerator systems. I.e., ultimately over $50 \%$ of the facility capital costs should be in beam-lines.

\section{ACCELERATOR DESIGN}

\subsection{Maturity of System and Component Design}

The past decade has also seen a steady evolution and maturity of accelerator design and technology used in synchrotron light sources. Lattice designs for low emittance beams are quite mature, with a variety of standard lattice configurations known.

The design of major components is also quite mature. Accurate design and manufacture of combined function magnets (e.g., curved gradient dipole magnets, quadrupole magnets with $\mathrm{x}-\mathrm{y}$ corrector windings, sextupole magnets with skew quadrupole windings, etc.) are becoming common. Superconducting RF cavity designs are also relatively mature and available commercially. Ultra-high-vacuum chambers for storage rings are also readily manufactured by several suppliers, and can achieve a vacuum of 1.0 nTorr or better with relative ease.

Another key technology that is now well developed is the design and manufacture of high quality undulators, using either pure permanent magnets or hybrid designs. While early undulators were usually operated on either the fundamental or low odd harmonics (third or fifth), new undulators can be designed, manufacture and accurately shimmed for operation on higher harmonics. For example, the small-gap undulator at CLS is being design to operate using up to the ninth harmonic, with eleventh harmonic operation also likely feasible. The impact of these newer designs cannot be underestimated, 
as they allow generation of hard x-rays at significantly lower electron beam energies.

\subsection{Maturity of Industrial Suppliers}

One other significant development in recent years is the growing capability of industry to design and supply major accelerator components or systems. As a result, newer light source facilities can use competitive design-build contracts for a larger portion of the facility construction, helping to reduce overall construction costs and internal laboratory design effort.

\subsection{Light Source Designs Evolution}

The user photon requirements in section 2, when combined with the present state of light source accelerator technology, provide the context for examining the direction of light source evolution. First, the user community wants undulator-based radiation, if possible, because of its high brightness and beam quality. In fact, bending magnet radiation is often viewed as so inferior that many users will not consider using it. For light source designers, this means that the number of straights available for insertion devices should be as large as possible, with a careful consideration of the optimum length to try to maximize the use of straights. The range and type of insertion devices that will be used on a storage ring should be known as early as possible to permit this optimization. Techniques for maximizing the use of a straight include multiple beam-lines from a wiggler source and using two undulators separated by a chicane in a single straight. The chicane introduces a small angular separation between the two beams from the undulators, which can be used to direct the beams to separate experiment end-stations.

The other major direction for future light source evolution is towards lower beam energy, provided that sufficiently high-energy x-rays can still be produced for those experiments that require them. The key technical development here is the progress on high quality undulators, operated at high harmonics. For a given lattice design, lower energy results in decreased emittance and increased beam current for the same RF power load. Reduced emittance and increased current can improve undulator performance at high harmonics, and may compensate for the lower beam energy. The net effect can be to retain adequate performance for high-energy $\mathrm{x}$ rays, while improving the source performance at lower photon energies. In particular, infrared performance is almost completely determined by the available opening angle and the total circulating beam current, and is essentially independent of the beam energy. Changes that increase the total current while maintaining beam stability are welcome to these users.

There are several limits on beam energy reduction, which include:

- $\quad$ Reduced undulator performance at high harmonics because of imperfections in the undulator fields;
- Lifetime limitations at lower energy and higher current; and

- Increased sensitivity to beam instabilities at higher current.

At present, with the typical range of desired photon energies, the optimum beam energy appears to be between 2.0 and $3.0 \mathrm{GeV}$. As a result, most new synchrotron light facilities proposed or under construction are in this energy range.

The other major constraint on new facilities is cost, leading to designs that reduce the overall facility cost per insertion device beam-line. Usually this takes the form of trying to minimize the length or fraction of the circumference that is used by ring magnets. This optimization leads to the increasing use of combined function magnets, reducing the number of independent components around the ring. The use of superconducting cavities for the main rf system can also decrease the number of straights not available for insertion devices.

\section{NEW LIGHT SOURCES}

Table 1 summarizes the number of light sources currently in operation, under construction, or proposed (see [1]). While past facilities covered a wide range of beam energy, the table also shows the growing number of facilities in the intermediate energy range between 1.5 and $3.5 \mathrm{GeV}$. Of the five intermediate energy rings under construction, it is interesting to examine two facilities, the Swiss Light Source and the Canadian Light Source, more closely.

Table 1: Synchrotron Light Sources (parentheses indicate parasitic operation)

\begin{tabular}{|c|ccc|}
\hline $\begin{array}{c}\text { Energy } \\
(\mathrm{GeV})\end{array}$ & $\mathrm{E}<1.5$ & $1.5<\mathrm{E}<3.5$ & $3.5<\mathrm{E}$ \\
\hline In Operation & $22(3)$ & $15(4)$ & $7(3)$ \\
$\begin{array}{c}\text { Under } \\
\text { Construction } \\
\text { Proposed }\end{array}$ & 8 & 5 & - \\
\hline
\end{tabular}

\subsection{Swiss Light Source}

The SLS is a $2.4 \mathrm{GeV}$ ring with a diameter of $288 \mathrm{~m}$, a natural emittance of $4.5 \mathrm{~nm}$ and a design maximum current of $400 \mathrm{~mA}$, which is just completing commissioning before starting routine operation in 2001 August (see [2]). This ring is designed with 12 straights in three different lengths - six $4 \mathrm{~m}$, three $7 \mathrm{~m}$, and three $11 \mathrm{~m}$ straights - to allow better optimization of the use of the straights. One long straight is used for injection, and two short straights are used for the main ring rf system, leaving ten straights with varied lengths for insertion devices. The SLS commissioning is notable because it included operation with a small-gap undulator, and it 
achieved the design current of $400 \mathrm{~mA}$ within six months of the initial stored beam.

\subsection{Canadian Light Source}

The CLS is a $2.9 \mathrm{GeV}$ ring with a circumference of $170 \mathrm{~m}$, a natural emittance of $18 \mathrm{~nm}$, and a design current of $500 \mathrm{~mA}$ (200 mA for initial operation). Construction started in 1999 July, and the present status is described in more detail in [3]. This facility has twelve $5.2 \mathrm{~m}$ straights, with one used for injection, one used for a single $500 \mathrm{MHz}$ superconducting rf cavity, and one currently reserved for ring diagnostic and feedback systems. Significantly, five of the seven initial beam-lines use insertion devices, with the two exceptions being infrared beam-lines. In addition, most of the proposed additional beam-lines also require insertion devices, leading the facility design group to set the goal of obtaining two beam-lines on each straight. To achieve this goal, straights using undulators are being designed to accommodate two chicaned devices, and straights using wigglers will have multiple beam-lines on a single wiggler. As well, the project construction team is making extensive use of design-build contracts for most accelerator and beam-line systems.

\subsection{Other New Sources}

Other new sources that have started construction, or are likely to start within the next year or two include:

- $\quad$ SPEAR 3 - an upgrade of the existing SSRL ring to a $3.0 \mathrm{GeV}$ third-generation design;

- BOOMERANG - a compact $2.9 \mathrm{GeV}$, 12-cell design similar to the CLS for Australia;

- DIAMOND - a large ( 500 m) 3.0 GeV, 24-cell design for the United Kingdom;

- $\quad$ LLS - a $2.5 \mathrm{GeV}, 12$-cell design for Spain;

- $\quad$ SOLEIL - a large $(\sim 370 \mathrm{~m}) 3.0 \mathrm{GeV}, 16$-cell design for France;

- A 3.0 GeV design for Shanghai, China.

\subsection{Future Directions}

As discussed in section 3, the main direction for most future general-purpose sources will be to energies between 2.0 and $3.0 \mathrm{GeV}$, representing the compromises between reduced costs, reduced emittance and reduced energy while delivering undulator radiation up to $\sim 20 \mathrm{keV}$ and wiggler radiation up to $\sim 40 \mathrm{keV}$. At the same time, some well-defined niches may develop for smaller narrow purpose, high current rings for infrared, visible, and vacuum ultraviolet applications. Design studies will also continue on alternates to the conventional electron storage ring, such as energy-recovery linacs and free electron lasers, for high energy, high brightness applications.

\section{CONCLUSION}

Synchrotron light sources are a major accelerator success story, with the research using these facilities having a major impact on all scientific fields, well beyond physics applications that initiated their development two to three decades ago. As a consequence there is a steady growing user base in all the sciences, leading to a continuing demand for new and better facilities, and continuing opportunities and challenges for accelerator designers.

Key design challenges that remain include maintaining or increasing the photon flux and/or brightness for individual beam-lines, while reducing the total beam power; increasing the integration of accelerator and beamline system design to permit better overall system optimization; increasing facility reliability, stability and flexibility; and working to reduce overall system costs for the same beam-line performance.

\section{REFERENCES}

[1] http://www-ssrl.slac.stanford.edu/sr_sources.html [2] Andreas Streun, "Commissioning of the Swiss Light Source", this conference.

[3] Les Dallin, "The Canadian Light Source: An Update", this conference. 